\title{
On the Zweier Sequence Spaces of Fuzzy Numbers
}

\author{
Mehmet Şengönül \\ Department of Mathematics, Faculty of Arts and Sciences, Nevşehir Hacı Bektaş Veli University, 2000 Evler Mah. Zübeyde Hanım \\ Cad., 50300 Nevşehir, Turkey \\ Correspondence should be addressed to Mehmet Şengönül; msengonul@yahoo.com
}

Received 27 December 2013; Revised 27 February 2014; Accepted 1 March 2014; Published 3 April 2014

Academic Editor: Hari M. Srivastava

Copyright (C) 2014 Mehmet Şengönül. This is an open access article distributed under the Creative Commons Attribution License, which permits unrestricted use, distribution, and reproduction in any medium, provided the original work is properly cited.

It was given a prototype constructing a new sequence space of fuzzy numbers by means of the matrix domain of a particular limitation method. That is we have constructed the Zweier sequence spaces of fuzzy numbers $\left[\ell_{\infty}(F)\right]_{Z^{\eta}},[c(F)]_{Z^{\eta}}$, and $\left[c_{0}(F)\right]_{Z^{\eta}}$ consisting of all sequences $u=\left(u_{k}\right)$ such that $Z^{\eta} u$ in the spaces $\ell_{\infty}(F), c(F)$, and $c_{0}(F)$, respectively. Also, we prove that $\left[\ell_{\infty}(F)\right]_{Z^{\eta}}$, $[c(F)]_{Z^{\eta}}$, and $\left[c_{0}(F)\right]_{Z^{\eta}}$ are linearly isomorphic to the spaces $\ell_{\infty}(F), c(F)$, and $c_{0}(F)$, respectively. Additionally, the $\alpha(r)-, \beta(r)-$, and $\gamma(r)$-duals of the spaces $\left[\ell_{\infty}(F)\right]_{Z^{\eta}},[c(F)]_{Z^{\eta}}$, and $\left[c_{0}(F)\right]_{Z^{\eta}}$ have been computed. Furthermore, two theorems concerning matrix map have been given.

\section{Introduction and Preliminaries}

Let suppose that $\mathbb{N}, \mathbb{R}$, and $E_{i}$ are the set of all positive integers, all real numbers, and all bounded and closed intervals on the real line $\mathbb{R}$; that is, $E_{i}=\left\{a=\left[a^{-}, a^{+}\right]: a^{-} \leq\right.$ $x \leq a^{+}, a^{-}$and $\left.a^{+} \in \mathbb{R}\right\}$, respectively. For $a, b \in E_{i}$, define

$$
d(a, b)=\max \left\{\left|a^{-}-b^{-}\right|,\left|a^{+}-b^{+}\right|\right\} .
$$

It can easily be seen that $d$ defines a metric on $E_{i}$ and the pair $\left(E_{i}, d\right)$ is a complete metric space [1]. Let $X$ be nonempty set. According to Zadeh, a fuzzy subset of $X$ is a nonempty subset $\{(x, u(x)): x \in X\}$ of $X \times[0,1]$ for some function $u: X \rightarrow$ $[0,1][2]$. Consider a function $u: \mathbb{R} \rightarrow[0,1]$ as a subset of a nonempty base space $\mathbb{R}$ and denote the family of all such functions or fuzzy sets by $E$. Let us suppose that the function $u$ satisfies the following properties:

(1) $u$ is normal; that is, there exists an $x_{0} \in \mathbb{R}$ such that $u\left(x_{0}\right)=1$

(2) $u$ is fuzzy convex; that is, for any $x, y \in \mathbb{R}$ and $\mu \in$ $[0,1], u[\mu x+(1-\mu) y] \geq \min \{u(x), u(y)\}$

(3) $u$ is upper semicontinuous;

(4) the closure of $\{x \in \mathbb{R}: u(x) \geq 0\}$, denoted by $u^{0}$, is compact.

Then the function $u$ is called a fuzzy number [9].
Properties (1)-(4) imply that for each $\alpha \in[0,1]$, the $\alpha$-cut set of the fuzzy number $u$ defined by $u(\alpha)=\{x \in \mathbb{R}: u(x) \geq$ $\alpha\}$ is in $E_{i}$; that is, $u(\alpha)=\left[u^{-}(\alpha), u^{+}(\alpha)\right]$ for each $\alpha \in[0,1]$. We denote the set of all fuzzy numbers by $F$.

Also, the following statements hold:

(5) $u^{-}(\alpha)$ is a bounded and nondecreasing left continuous function on $(0,1]$;

(6) $u^{+}(\alpha)$ is a bounded and nonincreasing left continuous function on $(0,1]$;

(7) the functions $u^{+}(\alpha)$ and $u^{-}(\alpha)$ are right continuous at the point $\alpha=0$;

(8) $u^{+}(\alpha) \geq u^{-}(\alpha)$.

Sometimes, the representation of fuzzy numbers with $\alpha$ cut sets is cause failures according to algebraic operations. For example, if $u$ is any fuzzy number, then $u-u=$ $\left[u^{-}(\alpha), u^{+}(\alpha)\right]-\left[u^{-}(\alpha), u^{+}(\alpha)\right]=\left[u^{-}(\alpha)-u^{+}(\alpha), u^{+}(\alpha)-\right.$ $\left.u^{-}(\alpha)\right]$ is not equal to fuzzy zero.

In this study, we have used another type representation of a fuzzy number to avoid this type algebraic failure which is used in $[3,4]$.

Furthermore, we know that shape similarity of the membership functions does not reflect the conception itself, but the context in which it is used. Whether a particular shape is suitable or not can be determined only in the context of 
a particular application. However, many applications are not overly sensitive to variations in the shape. In such cases, it is convenient to use a simple shape, such as the triangular shape of membership function.

For example, let us consider any triangular fuzzy number $u$.

If the function

$$
u(x)= \begin{cases}\frac{x-u^{1}}{u^{2}-u^{1}}, & x \in\left[u^{1}, u^{2}\right], \\ \frac{u^{3}-x}{u^{3}-u^{2}}, & x \in\left[u^{2}, u^{3}\right] \\ 0, & \text { otherwise }\end{cases}
$$

is the membership function of the triangular fuzzy number $u$, then the fuzzy number $u$ can represent the notation $u=$ $\left(u^{1}, u^{2}: 1, u^{3}\right)$. Clearly this representation is unique for $u(x)$ and on the contrary for every $u=u^{(1,2: 1,3)}$, there is an $u(x)$ which is unique. The triangular fuzzy numbers will be denoted shortly by $u=u^{(1,2: 1,3)}$ and the set of all triangular fuzzy numbers will be denoted by $F$; that is,

$$
F=\left\{u=u^{(1,2: 1,3)}: u^{1} \leq u^{2: 1} \leq u^{3} \text { and } u^{1}, u^{2: 1}, u^{3} \in \mathbb{R}\right\} .
$$

The $u^{1}, u^{2: 1}$, and $u^{3}$ are called first, middle, and end points of triangular fuzzy number, respectively. Also the notation $u^{2}: 1$ says to us that, the height of the fuzzy number $u$ is 1 at the $u^{2}$.

Let $u, v \in F$ and $\lambda \in \mathbb{R}$. The operations addition and scalar multiplication are defined on $F$ as follows:

$$
\begin{aligned}
u+v= & u^{(1,2,3)}+v^{(1,2,3)} \\
= & \left(u^{1}+v^{1}, u^{2}+v^{2}, u^{3}+v^{3}\right)=w^{(1,2,3)}=w, \\
& \lambda u^{(1,2,3)}= \begin{cases}\lambda u^{(1,2,3)} & \text { if } \lambda \geq 0, \\
\lambda u^{(3,2,1)} & \text { if } \lambda<0 .\end{cases}
\end{aligned}
$$

In fact, it is necessary to make some assumptions on the set of fuzzy numbers for constructing an algebraic structure. For example, we must redefine fuzzy identity elements according to addition and multiplication.

Let us suppose that $u^{1} \leq 0, u^{2}=0$, and $u^{3} \geq 0$. If we accept that any fuzzy number

$$
\overline{0}=\left(u^{1}, 0, u^{3}\right)
$$

is a fuzzy zero, then the difference $u^{(1,2,3)}-u^{(1,2,3)}=$ $u^{(1-3,2-2,3-1)}=\left(u^{1}-u^{3}, 0, u^{3}-u^{1}\right)=\overline{0}$ is acceptable fuzzy zero for us. Therefore, we say that the inverse of the fuzzy number $u^{(1,2,3)}$ is $-u^{(1,2,3)}=\left(-u^{3},-u^{2},-u^{1}\right)$, according to addition and the $-u^{(1,2,3)}$ determines a fuzzy number. With this idea, we can solve equations in the form $x^{(1,2,3)}+u^{(1,2,3)}=\overline{0}$ which may be given by inexact data.

If we sum fuzzy number $u$ with fuzzy zero, then the spread of fuzziness of $u$ may increase or decrease. This is normal because we are summing a "fuzzy number" and "fuzzy zero."
This situation is valid for fuzzy one; that is, if the $\overline{1}$ fuzzy is one, then spread of fuzziness of the products $\overline{1} u$ or $u \overline{1}$ will be increase or decrease. This is normal, too.

The "fuzzy number $a$ " can be denoted by the notation $\left(u^{1}, a, u^{3}\right)$ and $\max \left\{\left|u^{1}-a\right|,\left|a-u^{3}\right|\right\}$ should be less than 1 . Theoretically, the assumption " $\max \left\{\left|u^{1}-a\right|,\left|a-u^{3}\right|\right\}$ should be less than 1" that is not necessary but in the practical, it has to be. For example, the "approximately 5 " can be taken as $\overline{5}=$ $(-4,5,15)$ but in the applications, generally, "approximately 5 " is taken as $\overline{5}=(5-t, 5,5+t),(0<t<1)$. This choice is more accurate than $\overline{5}=(-4,5,15)$. We know that, generally, in the practical applications, the spread of fuzziness is not very large. It is fact that the set $F$ is not linear space according to algebraic operations sense of (4).

The second important matter is the topology on the set $F$. If we want construct a topology on, then we can use the metric defined by $\bar{d}: F \times F \rightarrow \mathbb{R}$,

$$
\bar{d}\left(u^{(1,2,3)}, v^{(1,2,3)}\right):=\max _{i=1,2,3}\left\{\left|u^{i}-v^{i}\right|\right\}
$$

We can easily show that the set $F$ is a complete metric space with the metric $\bar{d}$.

The function $f: \mathbb{N} \rightarrow F, k \rightarrow f(k):=u_{k}^{(1,2,3)}$ is called a sequence of fuzzy numbers and is represented by $\left(u_{k}\right)=$ $\left(u_{k}^{(1,2,3)}\right),[5]$.

Let us denote the set of all sequences of fuzzy numbers by $w(F)$; that is,

$$
w(F):=\left\{u=\left(u_{k}^{(1,2,3)}\right): u: \mathbb{N} \rightarrow F, u(k)=u_{k}^{(1,2,3)}\right\}
$$

where $u_{k}^{1} \leq u_{k}^{2} \leq u_{k}^{3}$ and $u_{k}^{1}, u_{k}^{2}, u_{k}^{3} \in \mathbb{R}$ for all $k \in \mathbb{N}$, [6]. The $u_{k}^{1}, u_{k}^{2}$, and $u_{k}^{3}$ are called first, middle, and end points of general term of sequences of triangular fuzzy numbers, respectively. If degree of membership at $u_{k}^{2}$ is equal to 1 , then $\left(u_{k}\right)$ is a sequence of fuzzy number and if it is not, then the sequence $\left(u_{k}\right)$ is a sequence of the fuzzy sets.

Each subspace of $w(F)$ is called a sequence space of fuzzy numbers. We will write $\ell_{\infty}(F), c(F)$, and $c_{0}(F)$ for the spaces of all bounded, convergent, and null sequences of fuzzy numbers, respectively; that is,

$$
\begin{gathered}
\ell_{\infty}(F)=\left\{u=\left(u_{k}^{(1,2,3)}\right) \in w(F):\right. \\
\left.\sup _{k \in \mathbb{N}} \bar{d}\left(u_{k}^{(1,2,3)}, \overline{0}\right)<\infty\right\}, \\
c(F)=\left\{u=\left(u_{k}^{(1,2,3)}\right) \in w(F): \lim _{k} \bar{d}\left(u_{k}^{(1,2,3)}, u_{0}^{(1,2,3)}\right)=0,\right. \\
\left.u_{0} \in F\right\},
\end{gathered}
$$




$$
\begin{gathered}
\mathcal{c}_{0}(F)=\left\{u=\left(u_{k}^{(1,2,3)}\right) \in w(F): \lim _{k} \bar{d}\left(u_{k}^{(1,2,3)}, \overline{0}\right)=0\right\} \\
\ell_{p}(F)=\left\{u=\left(u_{k}^{(1,2,3)}\right) \in w(F): \sum_{k} \bar{d}\left(u_{k}^{(1,2,3)}, \overline{0}\right)^{p}<\infty\right. \\
1 \leq p<\infty\} .
\end{gathered}
$$

In [7], Nanda has studied the spaces of bounded and convergent sequences of fuzzy numbers and shown that they are complete metric spaces with the metric $\widehat{d}(u, v)=$ $\sup _{k} \max \left\{\left|u_{k}^{-}(\alpha)-v_{k}^{-}(\alpha)\right|,\left|u_{k}^{+}(\alpha)-v_{k}^{+}(\alpha)\right|\right\},(\forall \alpha \in[0,1])$.

By using the metric $\widehat{d}$, many spaces of fuzzy sequences have been built and published in famous math journals. By reviewing the literature, one can reach them easily, (e. g., see, [8-13] and others).

Definition 1. Let $\lambda(F) \subset w(F)$ and algebraic operations on $\lambda(F)$ sense of $(4)$. The function $\|\cdot\|: \lambda(F) \rightarrow \mathbb{R}$. The function $\|\cdot\|$ is called module on the set $\lambda(F)$ if it has the following properties:
(9) $\|u\|=0 \Leftrightarrow u=\theta$,
(10) $\|\alpha u\|=|\alpha|\|u\|$,
(11) $\|u+v\| \leq\|u\|+\|v\|$.

If the function $\|\cdot\|: \lambda(F) \rightarrow \mathbb{R}$ satisfies (9)-(11) on $\lambda(F)$, then $\lambda(F)$ is called module sequence space of the fuzzy numbers. If $\lambda(F)$ is complete with respect to the module $\|\cdot\|$, then $\lambda(F)$ is called complete module sequence space of the fuzzy numbers.

By Definition 1, we have "Every normed space is a module space but converse is not true."

Let $\lambda(F)$ and $\mu(F)$ be two spaces of fuzzy valued sequences and let $A=\left(a_{n k}\right)$ be an infinite matrix of positive real numbers $a_{n k}$, where $n, k \in \mathbb{N}$. Then, we say that $A$ defines a real-matrix mapping from $\lambda(F)$ to $\mu(F)$, and we denote it by writing $A: \lambda(F) \rightarrow \mu(F)$, if for every sequence $u=$ $\left(u_{k}^{(1,2,3)}\right) \in \lambda(F)$, the sequence $A u=\left\{\left(A u^{(1,2,3)}\right)_{n}\right\}$ is in $\mu(F)$ where

$$
\begin{aligned}
\left(A u^{(1,2,3)}\right)_{n} & =\sum_{k} a_{n k}^{(1,1,1)} u_{k}^{(1,2,3)} \\
& =\left(\sum_{k} a_{n k}^{(1,1,1)} u_{k}^{1}, \sum_{k} a_{n k}^{(1,1,1)} u_{k}^{2}, \sum_{k} a_{n k}^{(1,1,1)} u_{k}^{3}\right),
\end{aligned}
$$

and the series $\sum_{k} a_{n k}^{(1,1,1)} u_{k}^{1}, \sum_{k} a_{n k}^{(1,1,1)} u_{k}^{2}, \sum_{k} a_{n k}^{(1,1,1)} u_{k}^{3}$ are convergent for all $n \in \mathbb{N}$. By $(\lambda(F): \mu(F))$, we denote the class of matrices $A$ such that $A: \lambda(F) \rightarrow \mu(F)$. Thus, $A \in(\lambda(F): \mu(F))$ if and only if the series on the right side of (9) are convergent for each $n \in \mathbb{N}$ and every $u \in \lambda(F)$; we have $A u=\left\{\left(A u^{(1,2,3)}\right)_{n}\right\}_{n \in \mathbb{N}} \in \mu(F)$ for all $u \in \lambda(F)$.

Let

$$
[\lambda(F)]_{A}=\left\{u=\left(u_{k}^{(1,2,3)}\right) \in w(F): A u^{(1,2,3)} \in \lambda(F)\right\},
$$

where $\lambda(F)$ is a sequence space of fuzzy numbers. Then, the set $[\lambda(F)]_{A}$ of sequences of fuzzy numbers is called the domain of an infinite matrix $A$ in $\lambda(F)$.

Let the infinite matrix $A=\left(a_{n k}\right)$ of positive real numbers be the Cesàro matrix of one order; that is,

$$
a_{n k}=\left\{\begin{array}{ll}
\frac{1}{n+1}, & 0 \leq k \leq n \\
0, & \text { otherwise }
\end{array}(n, k \in \mathbb{N})\right.
$$

Then the $A$-transform of the sequence $\left(u \_k\right)=$ $((-2,-1,0),(0,1,2),(-2,-1,0),(0,1,2), \ldots,(-2,-1,0),(0,1$, $2), \ldots)$ of fuzzy numbers is equal to $((-1,0,1))$ which means that we can obtain a limit with this way. From here, we realize that any limitation method can transform sequences of fuzzy numbers into set of all sequences of fuzzy numbers. It is also very important to construct new sequence space of fuzzy numbers.

The idea to construct a new sequence space of real or complex numbers using by matrix domain of a particular limitation method has been employed many authors; for example, you can see Altay et al. [14], Başar and Altay [15], Malkowsky [16], $\mathrm{Ng}$ and Lee [17], and Wang [18]. They introduced the sequence spaces $\left(\ell_{p}\right)_{R_{t}}=e_{p}^{r}$ with $1 \leq p \leq \infty$, $\left(\ell_{\infty}\right)_{R_{t}}=r_{\infty}^{t}, c_{R_{t}}=r_{c}^{t}$ and $\left(c_{0}\right)_{R^{t}}=r_{0}^{t}$ in [19], $\left(\ell_{p}\right)_{\Delta}=b v_{p}$ in [15], $\left(\ell_{p}\right)_{C}=X_{p}$ in [17], and $\left(\ell_{p}\right)_{N^{q}}$ in [18], where $E^{r}, R^{t}$, $\Delta, C$, and $N_{q}$ denote the Euler, means order $r$, Riesz means with respect to the sequence $t=\left(t_{k}\right)$, backward difference matrix, Cesàro means of order one, and Nörlund means with respect to the sequence $q=\left(q_{k}\right)$, respectively, and $1 \leq p \leq$ $\infty$. In the present paper, following [14-18], we will define matrix domain of sequence spaces of fuzzy numbers and introduce the sequence spaces of fuzzy numbers $[c(F)]_{Z^{\eta}}$ and $\left[c_{0}(F)\right]_{Z^{\eta}}$ and derive some results related to those sequence spaces. Furthermore, we have computed the $\alpha(r)-, \beta(r)$ and $\gamma(r)$-duals of the spaces $[c(F)]_{Z^{\eta}}$ and $\left[c_{0}(F)\right]_{Z^{\eta}}$. Latter, we have characterized the matrix mappings from the spaces $[c(F)]_{Z^{\eta}}$ and $\left[c_{0}(F)\right]_{Z^{\eta}}$ to $\mu(F)$ and from $\mu(F)$ to $[c(F)]_{Z^{\eta}}$ and $\left[c_{0}(F)\right]_{Z^{\eta}}$ by employing the suitable relations between the related matrix classes, where $\mu(F)$ is a given sequence space. Now we will give a lemma.

Lemma 2. The sets $c(F), c_{0}(F)$, and $\ell_{\infty}(F)$ are complete module spaces with the module defined by

$$
\|u\|=\sup _{k} \max _{i=1,2,3}\left\{\left|u_{k}^{(i)}\right|\right\} .
$$

Proof. We consider only $\ell_{\infty}(F)$. It is easy to see that $\|\cdot\|$ is a module on $\ell_{\infty}(F)$. To show that $\ell_{\infty}(F)$ is complete in this module, suppose that $\left(u_{k}^{(i, n)}\right)=\left(\left(u_{k}^{(1, n)}, u_{k}^{(2, n)}, u_{k}^{(3, n)}\right)\right)$ is a fundamental sequence in $\ell_{\infty}(F)$ for each $n$. Then we have

$$
\epsilon>\left\|u_{k}^{(i, n)}-u_{k}^{(i, m)}\right\|=\sup _{k} \max _{i=1,2,3}\left\{\left|u_{k}^{(i, n)}-u_{k}^{(i, m)}\right|\right\}
$$

Hence we can write

$$
\epsilon>\left|u_{k}^{(i, n)}-u_{k}^{(i, m)}\right|, \quad i=1,2,3
$$


From here, we realize that $\left(u_{k}^{(1, n)}\right),\left(u_{k}^{(2, n)}\right)$, and $\left(u_{k}^{(3, n)}\right)$ are fundamental sequences of real numbers in $\mathbb{R}$. Since $\mathbb{R}$ is complete, the sequences $\left(u_{k}^{(1, n)}\right),\left(u_{k}^{(2, n)}\right)$, and $\left(u_{k}^{(3, n)}\right)$ are convergent in $\mathbb{R}$ for all $n \in \mathbb{N}$.

Let us suppose that $\lim _{n} u_{k}^{(i, n)}=u_{k}^{(i, 0)}$ for each $i=1,2,3$ and $k \in \mathbb{N}$. Since

$$
\sup _{k} \max _{i=1,2,3}\left\{\left|u_{k}^{(i, n)}-u_{k}^{(i, m)}\right|\right\}<\epsilon
$$

for all $n, m \geq k$, we have

$$
\begin{aligned}
\lim _{m \rightarrow \infty} \sup _{k} \max _{i=1,2,3}\left\{\left|u_{k}^{(i, n)}-u_{k}^{(i, m)}\right|\right\} \\
=\sup _{k} \max _{i=1,2,3}\left\{\left|u_{k}^{(i, n)}-u_{k}^{(i, 0)}\right|\right\}<\epsilon .
\end{aligned}
$$

This means that, for $i=1,2,3, u_{k}^{(i, n)} \rightarrow u_{k}^{(i, 0)}$ as $(n \rightarrow \infty)$; that is, $\lim _{n} u_{k}^{n}=u_{k}^{0}$ in $\left[\ell_{\infty}(F)\right]_{Z^{\eta}}$. On the other hand, since

$$
\begin{aligned}
\left\|u_{k}^{i, 0}\right\| & \leq \sup _{k} \max _{i=1,2,3}\left\{\left|u_{k}^{(i, 0)}-u_{k}^{(i, n)}\right|\right\}+\sup _{k} \max _{i=1,2,3}\left\{\left|u_{k}^{(i, n)}\right|\right\} \\
& =\left\|u_{k}^{i, 0}-u_{k}^{(i, n)}\right\|+\left\|u_{k}^{(i, n)}\right\|
\end{aligned}
$$

we realize that $\left(u_{k}^{i, 0}\right) \in \ell_{\infty}(F)$.

\section{The Zweier Sequence Spaces $\left[\ell_{\infty}(F)\right]_{Z^{\eta}}$, $[c(F)]_{Z^{\eta}}$, and $\left[c_{0}(F)\right]_{Z^{\eta}}$}

In this section, we wish to introduce the $\left[\ell_{\infty}(F)\right]_{Z^{\eta}},[c(F)]_{Z^{\eta}}$, and $\left[c_{0}(F)\right]_{Z^{\eta}}$ spaces, as the set of all sequences such that $Z^{\eta}$ transforms of them are in the spaces $\ell_{\infty}(F), c(F)$, and $c_{0}(F)$, respectively; that is

$$
\begin{gathered}
{\left[\ell_{\infty}(F)\right]_{Z^{\eta}}=\left\{\left(u_{k}^{(1,2,3)}\right) \in w(F): Z^{\eta} u^{(1,2,3)} \in \ell_{\infty}(F)\right\},} \\
{[c(F)]_{Z^{\eta}}=\left\{\left(u_{k}^{(1,2,3)}\right) \in w(F): Z^{\eta} u^{(1,2,3)} \in c(F)\right\},} \\
{\left[c_{0}(F)\right]_{Z^{\eta}}=\left\{\left(u_{k}^{(1,2,3)}\right) \in w(F): Z^{\eta} u^{(1,2,3)} \in c_{0}(F)\right\} .}
\end{gathered}
$$

Define the sequence of fuzzy numbers $v=\left(v_{k}^{(1,2,3)}\right)$ which will be frequently used, as the $Z^{\eta}$-transform of a sequence of fuzzy numbers $u=\left(u_{k}^{(1,2,3)}\right)$; that is,

$$
v_{k}^{(1,2,3)}=\eta u_{k}^{(1,2,3)}+(1-\eta) u_{k-1}^{(1,2,3)},
$$

where $u_{-1}=0,0<\eta<1$ and the matrix $Z^{\eta}=\left(z_{i k}\right)$ defined by

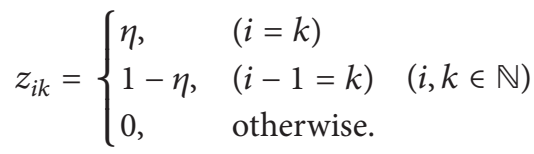

As we said above in (5), if we assume that each fuzzy number in the form $\left(u^{1}, 0, u^{3}\right)$ is a fuzzy zero, then $u_{k}^{(1,2,3)}$ can be left alone in equality (19). This assumption is important for our idea, which is always used in this paper.

Now, we may begin with the following theorem which is essential in the text.
Theorem 3. The sequence spaces $\left[\ell_{\infty}(F)\right]_{Z^{\eta}},[c(F)]_{Z^{\eta}}$, and $\left[c_{0}(F)\right]_{Z^{\eta}}$ are linearly isomorphic to the spaces $\ell_{\infty}(F), c(F)$, and $c_{0}(F)$, respectively; that is, $\left[\ell_{\infty}(F)\right]_{Z^{\eta}} \cong \ell_{\infty}(F),[c(F)]_{Z^{\eta}} \cong$ $c(F)$, and $\left[c_{0}(F)\right]_{Z^{\eta}} \cong c_{0}(F)$.

Proof. We will only consider $\left[\ell_{\infty}(F)\right]_{Z^{\eta}}$ with $\ell_{\infty}(F)$. To prove this, we should show the existence of a linear isometry between the spaces $\left[\ell_{\infty}(F)\right]_{Z^{\eta}}$ and $\ell_{\infty}(F)$. Consider the transformation defined $T$, with the notation of (19), from $\left[\ell_{\infty}(F)\right]_{Z^{\eta}}$ to $\ell_{\infty}(F)$ by $u \mapsto v=T u=\eta u_{i}^{(1,2,3)}+(1-\eta) u_{i-1}^{(1,2,3)}$. Equality $T(u+v)=T u+T v$ is clear. Let us suppose that $\alpha \geq 0$; then,

$$
\begin{aligned}
T(\alpha u) & =T\left(\alpha u_{i}^{(1,2,3)}\right)=\eta\left(\alpha u_{i}^{(1,2,3)}\right)+(1-\eta)\left(\alpha u_{i-1}^{(1,2,3)}\right) \\
& =\alpha\left[\eta u_{i}^{(1,2,3)}+(1-\eta) u_{i-1}^{(1,2,3)}\right]=\alpha T u .
\end{aligned}
$$

If $\alpha<0$, then

$$
\begin{aligned}
T(\alpha u) & =T\left(\alpha u_{i}^{(1,2,3)}\right)=\eta\left(\alpha u_{i}^{(3,2,1)}\right)+(1-\eta)\left(\alpha u_{i-1}^{(3,2,1)}\right) \\
& =\alpha\left[\eta u_{i}^{(1,2,3)}+(1-\eta) u_{i-1}^{(1,2,3)}\right]=\alpha T u ;
\end{aligned}
$$

that is, $T$ have the property homogeneity. Thus, $T$ is linear.

Let $v \in \ell_{\infty}(F), \mathscr{B}^{k}=\sum_{j=0}^{k}(-1)^{k-j}\left((1-\eta)^{k-j} / \eta^{k-j+1}\right)$, $\mathscr{B}^{k-1}=\sum_{j=0}^{k-1}(-1)^{k-j}\left((1-\eta)^{k-j} / \eta^{k-j+1}\right)$ and define the sequence $u=\left(u_{k}^{(1,2,3)}\right)$ by

$$
\left(\mathscr{B}^{k} v_{j}^{(1,2,3)}\right) .
$$

Then, we have

$$
\begin{aligned}
& \|u\|_{\left[\ell_{\infty}(F)\right]_{Z^{\eta}}} \\
& =\sup _{k \in \mathbb{N}} \bar{d}\left(Z^{\eta} u_{k}^{(1,2,3)}, \overline{0}\right) \\
& =\sup _{k} \bar{d}\left(\eta u_{k}^{(1,2,3)}+(1-\eta) u_{k-1}^{(1,2,3)}, \overline{0}\right) \\
& =\sup _{k \in \mathbb{N}} \bar{d}\left(\eta\left[\mathscr{B}^{k} v_{j}^{(1,2,3)}\right]+(1-\eta)\left[\mathscr{B}^{k-1} v_{j}^{(1,2,3)}\right], \overline{0}\right) \\
& =\sup _{k \in \mathbb{N}} \bar{d}\left(v_{k}^{(1,2,3)}, \overline{0}\right)=\|v\|_{\ell_{\infty}(F) .}
\end{aligned}
$$

That is, $T$ is norm preserving. Consequently, the spaces $\left[\ell_{\infty}(F)\right]_{Z^{\eta}}$ and $\ell_{\infty}(F)$ are linearly isometric. It is clear here that if the spaces $\left[\ell_{\infty}(F)\right]_{Z^{\eta}}$ and $\ell_{\infty}(F)$ are, respectively, replaced by the spaces $[c(F)]_{Z^{\eta}}$ and $c(F),\left[c_{0}(F)\right]_{Z^{\eta}}$, and $c_{0}(F)$, then we obtain the fact that $[c(F)]_{Z^{\eta}} \cong c(F)$ and $\left[c_{0}(F)\right]_{Z^{\eta}} \cong$ $c_{0}(F)$. This completes the proof.

Theorem 4. The sets $[c(F)]_{Z^{\eta}},\left[c_{0}(F)\right]_{Z^{\eta}}$, and $\left[\ell_{\infty}(F)\right]_{Z^{\eta}}$ are complete module sequence space of the fuzzy numbers with the module defined by

$$
\|u\|=\sup _{k} \max _{i=1,2,3}\left\{\left|\left(Z^{\eta} u^{(i)}\right)_{k}\right|\right\} .
$$


Proof. It was seen that in Theorem 3 that the sequence spaces of fuzzy numbers $\left[\ell_{\infty}(F)\right]_{Z^{\eta}},[c(F)]_{Z^{\eta}}$, and $\left[c_{0}(F)\right]_{Z^{\eta}}$ are linearly isometric to the spaces $\ell_{\infty}(F), c(F)$, and $c_{0}(F)$, respectively. Also, since the matrix $Z^{\eta}$ is normal (see, [20]) and $\ell_{\infty}(F), c(F)$, and $c_{0}(F)$ are complete module spaces (see Lemma 2), we see that the sequence spaces $\left[\ell_{\infty}(F)\right]_{Z^{\eta}}$, $[c(F)]_{Z^{\eta}}$, and $\left[c_{0}(F)\right]_{Z^{\eta}}$ are complete module spaces with the module defined in (25).

Let $\left(\vartheta_{n}\right)$ be a sequence of nonnegative real numbers which are not all zero and $P_{n}=\vartheta_{0}+\vartheta_{1}+\cdots+\vartheta_{n}$, for all $n \in \mathbb{N}$. The matrix $\mathcal{N}_{\vartheta}=\left(p_{n k}\right)$ is defined by

$$
p_{n k}= \begin{cases}\frac{\vartheta_{n-k}}{P_{n}}, & \text { if } k \leq n, \\ 0, & \text { otherwise }\end{cases}
$$

that is called the Nörlund matrix.

Other one of the best known matrix is $R=\left(r_{n k}\right)$, the Riesz matrix which is a lower triangular matrix defined by

$$
r_{n k}= \begin{cases}\frac{\vartheta_{k}}{P_{n}}, & 0 \leq k \leq n, \\ 0, & k>n,\end{cases}
$$

for all $n, k \in \mathbb{N}$, where $\left(\vartheta_{k}\right)$ is real sequence with $\vartheta_{0}>0, \vartheta_{k} \geq 0$ [9].

Let $\left(u_{n}\right)$ be a sequence of fuzzy numbers. If $\bar{d}\left(u_{n}, u_{0}\right) \rightarrow$ 0 as $n \rightarrow \infty$, then $\bar{d}\left((\mathcal{N} u)_{n}, u_{0}\right) \rightarrow 0$ as $n \rightarrow$ $\infty$ and $\lim _{n}\left((R u)_{n}, u_{0}\right) \rightarrow 0$ as $n \rightarrow \infty$, (see [21, Theorem 3.1 and Theorem 3.2]). That is, the matrices $\mathcal{N}_{9}$ and $R$ preserve the limits on the set of all convergent sequences of fuzzy numbers.

Theorem 5. Let $\left(u_{n}\right)=\left(u_{n}^{(1,2,3)}\right)$ be a sequence of fuzzy numbers. If $\bar{d}\left(u_{n}^{(1,2,3)}, u_{0}^{(1,2,3)}\right) \rightarrow 0$ as $\rightarrow \infty$, then $\bar{d}\left(\eta u_{n}^{(1,2,3)}+\right.$ $\left.(1-\eta) u_{n-1}^{(1,2,3)}, u_{0}^{(1,2,3)}\right) \rightarrow 0$ as $n \rightarrow \infty$. That is, the matrix $Z^{\eta}$ is regular.

Proof. Let $\left(u_{n}\right)=\left(u_{n}^{(1,2,3)}\right)$ be a sequence of fuzzy numbers and $\bar{d}\left(u_{n}^{(1,2,3)}, u_{0}^{(1,2,3)}\right) \rightarrow 0$ as $n \rightarrow \infty$. Then for a given $\epsilon>0$, there exists a positive integer $n_{0}$ such that

$$
\bar{d}\left(u_{n}^{(1,2,3)}, u_{0}^{(1,2,3)}\right)<\frac{\epsilon}{2 M} \text { for } n \geq n_{0} .
$$

Thus we can write

$$
\begin{aligned}
& \bar{d}\left(\left(Z^{\eta} u^{(1,2,3)}\right)_{n}, u_{0}^{(1,2,3)}\right) \\
&= \bar{d}\left(\eta u_{n}^{(1,2,3)}+(1-\eta) u_{n-1}^{(1,2,3)}, u_{0}^{(1,2,3)}\right) \\
& \quad \leq \eta \bar{d}\left(u_{n}^{(1,2,3)}, u_{0}^{(1,2,3)}\right)+(1-\eta) \bar{d}\left(u_{n-1}^{(1,2,3)}, u_{0}^{(1,2,3)}\right) .
\end{aligned}
$$

If we take $=\max \{\eta,(1-\eta)\}$, then we see that $\bar{d}\left(\left(Z^{\eta} u^{(1,2,3)}\right)_{n}\right.$, $\left.u_{0}\right)<\epsilon$. That is

$$
\lim _{n}\left(\eta u_{n}^{(1,2,3)}+(1-\eta) u_{n-1}^{(1,2,3)}\right)=u_{0}^{(1,2,3)} .
$$

Theorem 6. The inclusions $c(F) \subset[c(F)]_{Z^{\eta}}$ and $c_{0}(F) \quad \subset$ $\left[c_{0}(F)\right]_{Z^{\eta}}$ strictly hold.

Proof. To prove the validity of the inclusion $c_{0}(F) \subset\left[c_{0}(F)\right]_{Z^{\eta}}$, let us take any $v \in c_{0}(F)$. Then, bearing in mind the regularity of the method $Z^{\eta}$ (see, Theorem 5), we immediately observe that $Z^{\eta} v \in c_{0}(F)$ which means that $v \in\left[c_{0}(F)\right]_{Z^{\eta}}$. Hence, the inclusion $c_{0}(F) \subset\left[c_{0}(F)\right]_{Z^{\eta}}$ holds. Furthermore, let us consider the sequence $u=\left(u_{k}^{(1,2,3)}\right)$ defined by $u_{k}^{(1,2,3)}=(-1)^{k}((1-$ $\left.\eta)^{k} / \eta^{k+1}\right)((k-1) / k, 3 / 2,(3 k-1) / k)$ for all $k \in \mathbb{N}$. Then, we have $\left(Z^{\eta} u^{(1,2,3)}\right)_{k}=(-((1-\eta) / \eta)(0,3 / 2,2), \ldots, \overline{0}, \overline{0}, \ldots)$ which implies that $u$ is in $\left[c_{0}(F)\right]_{Z^{\eta}}$ but $u \notin c_{0}(F)$. This shows that the inclusion is strict. One can see by analogy that the strict inclusion $c(F) \subset[c(F)]_{Z^{\eta}}$ also holds. This completes the proof.

Let $(F), \mu(F) \subset w(F)$, and $A=\left(a_{n k}\right)$ be an infinite matrix of fuzzy numbers and consider following expressions:

$$
\begin{gathered}
\sup _{n \in \mathbb{N}} \sum_{k} \bar{d}\left(a_{n k}, \overline{0}\right)<\infty, \\
\sup _{n \in \mathbb{N}} \sum_{k}\left[\bar{d}\left(a_{n k}, \overline{0}\right)\right]^{q}<\infty, \\
\lim _{n} \bar{d}\left(a_{n k}, \alpha_{k}\right)=0, \quad \text { where } \alpha_{k} \in w(F,) \\
\lim _{n} \sum_{k} \bar{d}\left(a_{n k}, \overline{0}\right)=0, \\
\lim _{n} \sum_{k} a_{n k}=\overline{1}, \\
\lim _{n} a_{n k}=\overline{0}, \quad k \in \mathbb{N} .
\end{gathered}
$$

In [9], some matrix classes are characterized by Talo and Başar which are given in the following lemma.

Lemma 7 (see [9]). Let us suppose that

(12) $A \in\left(\ell_{\infty}(F): \ell_{\infty}(F)\right), A \in\left(c(F): \ell_{\infty}(F)\right), A \in$ $\left(c_{0}(F): \ell_{\infty}(F)\right)$ if and only if (31) holds,

(13) $A \in\left(\ell_{\infty}(F): c_{0}(F)\right)$ if and only if (34) holds,

(14) $A \in\left(c_{0}(F): c(F)\right)$ if and only if (31) and (33) hold,

(15) $A \in\left(c_{0}(F): c_{0}(F)\right)$ if and only if (31) and (33) also hold with $\alpha_{k}=\overline{0}$ for all $k \in \mathbb{N}$,

(16) $A \in\left(\ell_{p}(F): c(F)\right)$ if and only if (32) and (33) hold,

(17) $A \in\left(\ell_{p}(F): c_{0}(F)\right)$ if and only if (32) and (33) also hold with $\alpha_{k}=\overline{0}$ for all $k \in \mathbb{N}$,

(18) $A \in(c(F): c(F), p)$ if and only if (31), (35), and (33) also hold with $\alpha_{k}=\overline{0}$ for all $k \in \mathbb{N}$.

Analogously to Talo and Başar, we can prove following proposition.

Proposition 8. $A \in(c(F): c(F))$ if and only if (31) and (33) also hold with $\alpha_{k}=\overline{0}$ for all $k \in \mathbb{N}$. 
Proposition 9. $A \in\left(c_{0}(F): \ell_{1}(F)\right)$ if and only if $\sup _{G} \sum_{k} \bar{d}\left(\sum_{n \in G} a_{n k}, \overline{0}\right)<\infty$.

We know that each $r \in \mathbb{R}$ can be considered as a fuzzy number in the form of

$$
\bar{r}(t)= \begin{cases}1, & \text { if } t=r \\ 0, & \text { if } t \neq r\end{cases}
$$

Therefore, if $A=\left(a_{n k}\right)$ is a matrix of real numbers, the conditions in Lemma 7 and Propositions 8 and 9 are still valid.

\section{Real Duals of the Spaces $\left[\ell_{\infty}(F)\right]_{Z^{\eta}},[c(F)]_{Z^{\eta}}$, and $\left[c_{0}(F)\right]_{Z^{\eta}}$}

In this section, we state and prove the theorems determining the $\alpha(r)-, \beta(r)$-, and $\gamma(r)$-duals of the spaces $\left[\ell_{\infty}(F)\right]_{Z^{n}}$, $[c(F)]_{Z^{n}}$, and $\left[\mathcal{c}_{0}(F)\right]_{Z^{\eta}}$. For the sequence spaces $\lambda(F)$ and $\mu(F)$, define the set $S(\lambda(F), \mu(F))$ by

$$
\begin{aligned}
S( & \lambda(F), \mu(F)) \\
\quad & =\left\{a^{(1,1,1)} \in w(\mathbb{R}):\left(a_{k} x_{k}^{(1,2,3)}\right) \in \mu(F) \forall x \in \lambda(F)\right\},
\end{aligned}
$$

where $w(\mathbb{R})$ denotes all real valued sequences space. With the notation of (38), the $\alpha(r)-, \beta(r)$-, and $\gamma(r)$-duals of a sequence space $\lambda(F)$, which are, respectively, denoted by $\lambda^{\alpha(r)}(F), \lambda^{\beta(r)}(F)$, and $\lambda^{\gamma(r)}(F)$, are defined by

$$
\begin{aligned}
& \lambda^{\alpha(r)}(F)=S\left(\lambda(F), \ell_{1}(F)\right), \\
& \lambda^{\beta(r)}(F)=S(\lambda(F), c s(F)), \\
& \lambda^{\gamma(r)}(F)=S(\lambda(F), b s(F)) .
\end{aligned}
$$

We will use a technique, in the proof of the Theorems 10 and 13 , which is used in $[14,15,22]$.

Theorem 10. The $\gamma(r)$-dual of the spaces $\left[\ell_{\infty}(F)\right]_{Z^{n}},[c(F)]_{Z^{n}}$, and $\left[c_{0}(F)\right]_{Z^{n}}$ is the set

$$
\begin{aligned}
D_{1}= & \left\{a^{(1,1,1)} \in w(\mathbb{R}):\right. \\
& \left.\sup _{n \in \mathbb{N}} \sum_{i=0}^{n} \bar{d}\left(\sum_{j=i}^{n}(-1)^{n-j} \frac{(1-\eta)^{n-j}}{\eta^{n-j+1}} a_{n-j}^{(1,1,1)}, \overline{0}\right)<\infty\right\} .
\end{aligned}
$$

Proof. Since the proof is similar for the rest of the spaces, we determine only $\gamma(r)$-dual of the set $\left[c_{0}(F)\right]_{Z^{\eta}}$. Let $a^{(1,1,1)} \in$
$w(\mathbb{R})$ and define the matrix $T=\left(t_{i j}^{\eta}\right)$ via the sequence $a=$ $\left(a_{i}^{(1,1,1)}\right)$ by

$$
\begin{aligned}
& t_{i j}^{\eta} \\
& = \begin{cases}\sum_{j=i}^{n}(-1)^{n-j} \frac{(1-\eta)^{n-j}}{\eta^{n-j+1}} a_{n-j}^{(1,1,1)} & (0 \leq i \leq n) \quad(i, j, n \in \mathbb{N}) \\
0, & (i>n) .\end{cases}
\end{aligned}
$$

Bearing in mind relation (19), we immediately derive that

$$
\begin{aligned}
& \sum_{i=0}^{n} a_{i}^{(1,1,1)} x_{i}^{(1,2,3)} \\
& \quad=\sum_{i=0}^{n} \sum_{j=i}^{n}(-1)^{n-j} \frac{(1-\eta)^{n-j}}{\eta^{n-j+1}} a_{n-j}^{(1,1,1)} y_{i}^{(1,2,3)}=\left(T y^{(1,2,3)}\right)_{n}
\end{aligned}
$$

From (42), we realize that $a x=\left(a_{i}^{(1,1,1)} x_{i}^{(1,2,3)}\right) \in b s(F)$ whenever $x \in\left[c_{0}(F)\right]_{Z^{\eta}}$ if and only if $T y^{(1,2,3)} \in \ell_{\infty}(F)$ whenever $y^{(1,2,3)} \in \mathcal{c}_{0}(F)$. Then, we derive by Part (12) of Lemma 7 , and we have

$$
\sup _{n \in \mathbb{N}} \sum_{i=0}^{n} \bar{d}\left(\sum_{j=i}^{n}(-1)^{n-j} \frac{(1-\eta)^{n-j}}{\eta^{n-j+1}} a_{n-j}^{(1,1,1)}, \overline{0}\right)<\infty
$$

which yields the consequence that $\left[c_{0}(F)\right]_{Z^{\eta}}^{\gamma(r)}=D_{1}$.

Theorem 11. $\left[c_{0}(F)\right]_{Z^{\eta}}^{\gamma(r)}$ is the set $D_{2}$, where

$$
\begin{aligned}
D_{2}=\{a \in w(\mathbb{R}): & \\
& \sup _{G} \sum_{k} \bar{d} \\
& \times\left(\sum_{n \in G} \sum_{j=i}^{n}(-1)^{n-j}\right. \\
& \left.\left.\times \frac{(1-\eta)^{n-j}}{\eta^{n-j+1}} a_{n-j}^{(1,1,1)}, \overline{0}\right)<\infty\right\} .
\end{aligned}
$$

Proof. This is clear from Proposition 9. 
Theorem 12. Define the sets $D_{3}$ and $D_{4}$ by

$$
\begin{aligned}
D_{3}= & \left\{a^{(1,1,1)} \in w(\mathbb{R}):\right. \\
& \sup _{n \in \mathbb{N}} \sum_{i=0}^{n} \bar{d} \\
& \times\left(\sum_{j=i}^{n}(-1)^{n-j}\right. \\
D_{4}= & \left\{a^{(1,1,1)} \in w^{n}(\mathbb{R}):\right. \\
\lim _{n \rightarrow \infty} \sum_{i} \bar{d} & \left.\left.\eta_{n-j}^{(1,1,1)}, \overline{0}\right)<\infty\right\}, \\
\times\left(\sum_{j=i}^{n}(-1)^{n-j}\right. & \left.\left.\times \frac{(1-\eta)^{n-j}}{\eta^{n-j+1}} a_{n-j}^{(1,1,1)}, \overline{0}\right) \text { exists }\right\} .
\end{aligned}
$$

Then, $\left[c_{0}(F)\right]_{Z^{\eta}}^{\gamma(r)}=D_{3} \cap D_{4}$.

Proof. Consider

$$
\begin{aligned}
\sum_{i=0}^{n} a_{i}^{(1,1,1)} x_{i}^{(1,2,3)} & =\sum_{i=0}^{n} \sum_{j=i}^{n}(-1)^{n-j} \frac{(1-\eta)^{n-j}}{\eta^{n-j+1}} a_{n-j}^{(1,1,1)} y_{i}^{(1,2,3)} \\
& =\left(T y^{(1,2,3)}\right)_{i} ; \quad n \in \mathbb{N}
\end{aligned}
$$

where $T=\left(t_{i n}^{\eta}\right)$ is defined by

$$
\begin{aligned}
& t_{\text {in }}^{\eta} \\
& \quad=\left\{\begin{array}{ll}
\sum_{j=i}^{n}(-1)^{n-j} \frac{(1-\eta)^{n-j}}{\eta^{n-j+1}} a_{n-j}^{(1,1,1)}, & (0 \leq i \leq n) \\
0, & (i>n) .
\end{array} \quad(i, n \in \mathbb{N})\right.
\end{aligned}
$$

Thus, we deduce from Proposition 8 with (47) that $a x=$ $\left(a_{i}^{(1,1,1)} x_{i}^{(1,2,3)}\right) \in c s(F)$ whenever $x=\left(x_{i}^{(1,2,3)}\right) \in[c(F)]_{Z^{\eta}}$ if and only if $T y \in c(F)$ whenever $y=\left(y_{i}^{(1,2,3)}\right) \in c(F)$. It is obvious that the columns of that matrix $T$, defined by (47) are in the space $c(F)$. Therefore, we derive the consequences from Proposition 8 that $c^{\beta(r)}\left(Z^{\eta}, F\right)=D_{3} \cap D_{4}$.

Theorem 13. The $\gamma(r)$-dual of the space $[c(F)]_{Z^{\eta}}$ is the set $D_{3}$.

Proof. The proof of this theorem is similar to the proof of Theorem 10, so we omit it.

\section{Matrix Transformations}

For the first time, Lorentz introduced the concept of dual summability methods for the limitation which depends on a Stieltjes integral and passed to the discontinuous matrix methods by means of a suitable step function in [23]. Later, many authors such as Başar [24], Başar and Çolak [25], Kuttner [26], Lorentz and Zeller [27], and Şengönül and Başar [22] worked on the dual summability methods.

Let us suppose that the set $[\lambda(F)]_{Z^{\eta}}$ is any of the sets $\left[c_{0}(F)\right]_{Z^{\eta}}, c\left(Z^{\eta}, F\right)$, and $\left[\ell_{\infty}(F)\right]_{Z^{\eta}}$. In this section, we characterize the matrix mappings from $[\lambda(F)]_{Z^{\eta}}$ into any given sequence space of fuzzy numbers via the concept of the dual summability methods and vice versa, so we call it the Zweier dual summability methods.

Let us suppose that the sequences $u=\left(u_{i}^{(1,2,3)}\right)$ and $v=$ $\left(v_{i}^{(1,2,3)}\right)$ are connected with (19) and let the $A$-transform of the sequence $u=\left(u_{i}^{(1,2,3)}\right)$ be $z=\left(z_{i}^{(1,2,3)}\right)$ and let the $B$-transform of the sequence $v=\left(v_{i}^{(1,2,3)}\right)$ be $t=\left(t_{i}\right)$; that is,

$$
\begin{aligned}
& z_{i}^{(1,2,3)}=\left(A u^{(1,2,3)}\right)_{i}=\sum_{i} a_{n i}^{(1,1,1)} u_{i}^{(1,2,3)}, \quad(i \in \mathbb{N}), \\
& t_{i}^{(1,2,3)}=\left(B v^{(1,2,3)}\right)_{i}=\sum_{i} b_{n i}^{(1,1,1)} v_{i}^{(1,2,3)}, \quad(i \in \mathbb{N}) .
\end{aligned}
$$

It is clear here that the method $B$ is applied to the $Z^{\eta}$ transform of the sequence $u=\left(u_{i}^{(1,2,3)}\right)$ while the method $A$ is directly applied to the terms of the sequence $u=\left(u_{i}^{(1,2,3)}\right)$. Therefore the methods $A$ and $B$ are essentially different (see, [24]).

Let us assume the existence of the matrix product $B Z^{\eta}$ which is a much weaker assumption than the conditions on the matrix $B$ belonging to any matrix class, in general. If $z_{i}^{(1,2,3)}$ becomes $t_{i}^{(1,2,3)}$ (or $t_{i}^{(1,2,3)}$ becomes $z_{i}^{(1,2,3)}$ ), under the application of the formal summation by parts, then the methods $A$ and $B$ in (48) are called Zweier dual type matrices. This leads us to fact that $B Z^{\eta}$ exists and is equal to $A$ and $\left(B Z^{\eta}\right) u^{(1,2,3)}=B\left(Z^{\eta} u^{(1,2,3)}\right)$ formally holds. This statement is equivalent to the relation

$$
\begin{aligned}
& b_{n i}^{(1,1,1)}=\sum_{j=i}^{n}(-1)^{n-j} \frac{(1-\eta)^{n-j}}{\eta^{n-j+1}} a_{n, n-j}^{(1,1,1)} \\
& \text { or } \\
& a_{n k}^{(1,1,1)}=\eta b_{n k}^{(1,1,1)}+(1-\eta) b_{n, k+1}^{(1,1,1)}
\end{aligned}
$$

for all $n, k \in \mathbb{N}$.

Now we may give the following theorem concerning to the Zweier dual matrices.

Theorem 14. Let $A=\left(a_{n k}^{(1,1,1)}\right)$ and $B=\left(b_{n k}^{(1,1,1)}\right)$ be the Zweier dual type matrices, $\mu(F)$ any given sequence space, and $\left(a_{n k}^{(1,1,1)}\right)_{k \in \mathbb{N}} \in \ell_{1}(F)$. Then, $A \in\left([c(F)]_{Z^{\eta}}: \mu(F)\right)$ if and only if $B \in(c(F): \mu(F))$.

Proof. Suppose that $A=\left(a_{n k}^{(1,1,1)}\right)$ and $B=\left(b_{n k}^{(1,1,1)}\right)$ are Zweier dual type matrices, dual type matrices that is, to say that (49) 
holds and $\mu(F)$ is any given sequence space and take account that the spaces $[c(F)]_{Z^{\eta}}$ and $c(F)$ are linearly isomorphic.

Let $A \in\left([c(F)]_{Z^{\eta}}: \mu(F)\right)$ and take any $y \in c(F)$. Then, $B Z^{\eta}$ exists and $\left(a_{n k}^{(1,1,1)}\right)_{k \in \mathbb{N}} \in D_{2} \cap D_{3}$ which yields $\left(b_{n k}^{(1,1,1)}\right)_{k \in \mathbb{N}} \in \ell_{1}(F)$ for each $n \in \mathbb{N}$. Hence $B y^{(1,2,3)}$ exists for each $y \in c(F)$ and thus letting $m \rightarrow \infty$ in the equality

$$
\begin{aligned}
\sum_{i=0}^{m} b_{n i}^{(1,1,1)} y_{i}^{(1,2,3)} & \\
= & \sum_{i=0}^{m-1}\left[\eta b_{n i}^{(1,1,1)}+(1-\eta) b_{n, i+1}^{1,1,1)}\right] x_{i}^{(1,2,3)} \\
& +\eta b_{n m} x_{n}^{(1,1,1)}, \quad(n \in \mathbb{N}),
\end{aligned}
$$

we have, by (49), that $B y=A x$ which leads us to the consequence $B \in(c(F): \mu(F))$.

Conversely, suppose that $B \in\left([c(F)]_{Z^{\eta}}: \mu(F)\right)$ and take any $x \in[c(F)]_{Z^{\eta}}$. Then, $A x$ exists. Therefore, we obtain from the equality

$$
\begin{aligned}
\sum_{i=0}^{n} a_{n i}^{(1,1,1)} x_{i}^{(1,2,3)} & =\sum_{i=0}^{n} \sum_{j=i}^{n}(-1)^{n-j} \frac{(1-\eta)^{n-j}}{\eta^{n-j+1}} a_{n, n-j}^{(1,1,1)} y_{i}^{(1,2,3)} \\
& =\sum_{i=0}^{n} b_{n i}^{(1,1,1)} y_{i}^{(1,2,3)} ; \quad(n \in \mathbb{N}),
\end{aligned}
$$

as $n \rightarrow \infty$ that $A x=B y$ and this shows that $A \in\left([c(F)]_{Z^{n}}\right.$ : $\mu(F))$. This completes the proof.

Theorem 15. Suppose that the elements of the infinite matrices $D=\left(d_{n k}^{(1,1,1)}\right)$ and $E=\left(e_{n k}^{(1,1,1)}\right)$ are connected with the relation

$$
e_{n k}=\eta d_{n k}^{(1,1,1)}+(1-\eta) d_{n, k-1}^{(1,1,1)}, \quad(n, k \in \mathbb{N})
$$

and $\mu(F)$ is any given sequence space. Then, $D \in(\mu(F)$ : $\left.[c(F)]_{Z^{\eta}}\right)$ if and if only $E \in(\mu(F): c(F))$.

Proof. Let $x \in \mu(F)$ and consider the following equality with (50):

$$
\begin{gathered}
(1-\eta) \sum_{k=0}^{n} d_{n, k-1}^{(1,1,1)} x_{k-1}^{(1,2,3)}+\eta \sum_{k=0}^{n} d_{n k}^{(1,1,1)} x_{k}^{(1,2,3)} \\
=\sum_{k=0}^{n} e_{n k}^{(1,1,1)} x_{k}^{(1,2,3)}, \quad(n, k \in \mathbb{N})
\end{gathered}
$$

which yields as $n \rightarrow \infty$ that

$$
\|D x\|_{[c(F)]_{Z^{\eta}}}=\|E x\|_{c(F)} .
$$

Now, we immediately have, by (54), that $D x \in[c(F)]_{Z^{\eta}}$ whenever $x \in \mu(F)$ if and if only Ex $\in c(F)$ whenever $x \in \mu(F)$ and this step completes the proof.

Now, right here, we give the following propositions which are obtained from Lemma 7 and Theorems 14 and 15.
Proposition 16. Let $A=\left(a_{n k}^{(1,1,1)}\right)$ be an infinite matrix of real numbers. Then,

$$
\begin{aligned}
& \text { (1) } A=\left(a_{n k}^{(1,1,1)}\right) \in\left(\left[\ell_{\infty}(F)\right]_{Z^{\eta}}: \ell_{\infty}(F)\right) \text { if and only if } \\
& \sup _{n \in \mathbb{N}} \sum_{k} \bar{d}\left(\sum_{j=i}^{n}(-1)^{n-j} \frac{(1-\eta)^{n-j}}{\eta^{n-j+1}} a_{n-j, k}^{(1,1,1)}, \overline{0}\right)<\infty,
\end{aligned}
$$

(2) $A=\left(a_{n k}^{(1,1,1)}\right) \in\left([c(F)]_{Z^{\eta}}: c(F)\right)$ if and only if (55) and

$$
\lim _{n} \bar{d}\left(\sum_{j=1}^{n}(-1)^{n-j} \frac{(1-\eta)^{n-j}}{\eta^{n-j+1}} a_{n-j, k}^{(1,1,1)}, \overline{0}\right)=0, \quad \forall k \in \mathbb{N}
$$

Proposition 17. Let $A=\left(a_{n k}^{(1,1,1)}\right)$ be infinite matrix real numbers. Then, $A=\left(a_{n k}^{(1,1,1)}\right) \in\left(\ell_{\infty}(F):\left[\ell_{\infty}(F)\right]_{Z^{\eta}}\right)$ if and only if

$$
\sup _{n \in \mathbb{N}} \sum_{k} \bar{d}\left(\eta a_{n k}^{(1,1,1)}+(1-\eta) a_{n, k-1}^{(1,1,1)}, \overline{0}\right)<\infty
$$

Proposition 18. Let $A=\left(a_{n k}^{(1,1,1)}\right)$ be an infinite matrix of real numbers. Then, $A=\left(a_{n k}^{(1,1,1)}\right) \in\left([c(F)]_{Z^{\eta}}: c(F)\right)$ if and only if (57) and

$$
\begin{array}{r}
\lim _{n} \bar{d}\left(\eta a_{n k}^{(1,1,1)}+(1-\eta) a_{n, k-1}^{(1,1,1)}, \alpha_{k}^{(1,2,3)}\right)=0, \\
\text { with } \alpha_{k}^{(1,2,3)} \in F, \quad k \in \mathbb{N} .
\end{array}
$$

\section{Disclosure}

All praise is due to Allah who gave us to information and I wish, the idea of this paper will be guide to new research with related area.

\section{Conflict of Interests}

The author declares that there is no conflict of interests regarding the publication of this paper.

\section{Acknowledgment}

The author would like to thank referee for his/her much encouragement, support, constructive criticism, careful reading and making a useful comment which improved the presentation and the readability of the paper.

\section{References}

[1] R. E. Moore, "Automatic error analysis in digital computation," Tech. Rep. LSMD-48421, Lockheed Missiles and Space Company, 1959.

[2] P. Diamond and P. Kloeden, Metric Spaces of Fuzzy Sets, World Scientific, River Edge, NJ, USA, 1994.

[3] D. Filev and R. Yager, "A generalized defuzzification method under BAD distributions," International Journal of Intelligent Systems, vol. 6, pp. 689-697, 1991. 
[4] M. Sugeno, "An introductory survey of fuzzy control," Information Sciences, vol. 36, no. 1-2, pp. 59-83, 1985.

[5] Z. Mitrovic and S. Rusov, "Z similarity measure among fuzzy sets," FME Transactions, vol. 34, pp. 115-119, 2006.

[6] Z. Zararsiz and M. Sengönül, Center of Gravity of Sequence Space of Fuzzy Numbers, AFMI, 2013.

[7] S. Nanda, "On sequence spaces of fuzzy numbers," Fuzzy Sets and Systems, vol. 33, pp. 123-126, 1989.

[8] H. Altınok, R. Çolak, and M. Et, " $\lambda$-difference sequence spaces of fuzzy numbers," Fuzzy Sets and Systems, vol. 160, no. 21, pp. 3128-3139, 2009.

[9] Ö. Talo and F. Başar, "Determination of the duals of classical sets of sequences of fuzzy numbers and related matrix transformations," Computers \& Mathematics with Applications, vol. 58, no. 4, pp. 717-733, 2009.

[10] B. C. Tripathy and A. J. Dutta, "Statistically convergent and Cesàro summable double sequences of fuzzy real numbers," Soochow Journal of Mathematics, vol. 33, no. 4, pp. 835-848, 2007.

[11] T. Bilgin, " $\Delta$-statistical and strong $\Delta$-Cesaro convergence of sequences of fuzzy numbers," Mathematical Communications, vol. 8, no. 1, pp. 95-100, 2003.

[12] Y. Altın, M. Mursaleen, and H. Altınok, "Statistical summability $(C, 1)$ for sequences of fuzzy real numbers and a Tauberian theorem," Journal of Intelligent \& Fuzzy Systems, vol. 21, no. 6, pp. 379-384, 2010.

[13] R. Colak, Y. Altın, and M. Mursaleen, "On some sets of difference sequences of fuzzy numbers," Soft Computing, vol. 15, pp. 787-793, 2011.

[14] B. Altay, F. Başar, and M. Mursaleen, "On the Euler sequence spaces which include the spaces $l_{p}$ and $l_{\infty}$. I," Information Sciences, vol. 176, no. 10, pp. 1450-1462, 2006.

[15] F. Başar and B. Altay, "On the space of sequences of $p$ bounded variation and related matrix mappings," Ukrainian Mathematical Journal, vol. 55, no. 1, pp. 108-118, 2003.

[16] E. Malkowsky, "Recent results in the theory of matrix transformations in sequence spaces," Matematichki Vesnik, vol. 49, no. 3-4, pp. 187-196, 1997.

[17] P. N. Ng and P. Y. Lee, "Cesàro sequence spaces of non-absolute type," Commentationes Mathematicae. Prace Matematyczne, vol. 20, no. 2, pp. 429-433, 1978.

[18] C. S. Wang, "On Nörlund sequence spaces," Tamkang Journal of Mathematics, vol. 9, no. 2, pp. 269-274, 1978.

[19] B. Altay and F. Başar, "Some paranormed Riesz sequence spaces of non-absolute type," Southeast Asian Bulletin of Mathematics, vol. 30, no. 4, pp. 591-608, 2006.

[20] A. Wilansky, Summability Through Functional Analysis, vol. 85 of North-Holland Mathematics Studies, North-Holland, Amsterdam, The Netherlands, 1984.

[21] B. C. Tripathy and A. Baruah, "Nörlund and Riesz mean of sequences of fuzzy real numbers," Applied Mathematics Letters, vol. 23, no. 5, pp. 651-655, 2010.

[22] M. Şengönül and F. Başar, "Some new Cesàro sequence spaces of non-absolute type which include the spaces $c_{0}$ and $c$," Soochow Journal of Mathematics, vol. 31, no. 1, pp. 107-119, 2005.

[23] G. G. Lorentz, "Über Limitierungsverfahren, die von einem Stieltjes-Integral abhängen," Acta Mathematica, vol. 79, pp. 255272, 1947.

[24] F. Başar, "Matrix transformations between certain sequence spaces of $X_{p}$ and $l_{p}$," Soochow Journal of Mathematics, vol. 26, no. 2, pp. 191-204, 2000.
[25] F. Başar and R. Çolak, "Almost-conservative matrix transformations," Doğa, vol. 13, no. 3, pp. 91-100, 1989.

[26] B. Kuttner, "On dual summability methods," Mathematical Proceedings of the Cambridge Philosophical Society, vol. 71, pp. 67-73, 1972.

[27] G. G. Lorentz and K. Zeller, "Summation of sequences and summation of series," Proceedings of the Cambridge Philosophical Society, vol. 71, pp. 67-73, 1972. 


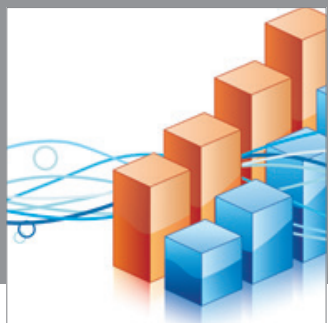

Advances in

Operations Research

mansans

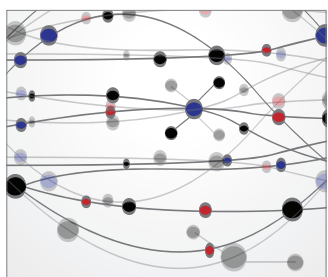

The Scientific World Journal
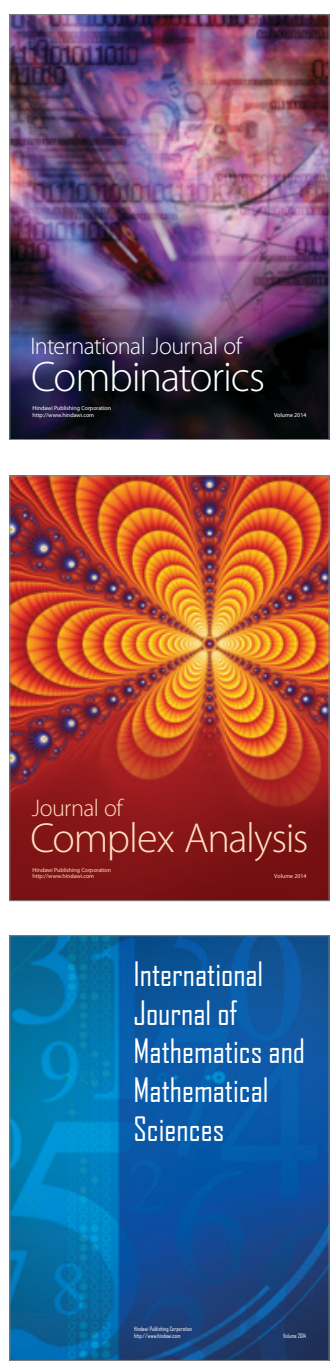
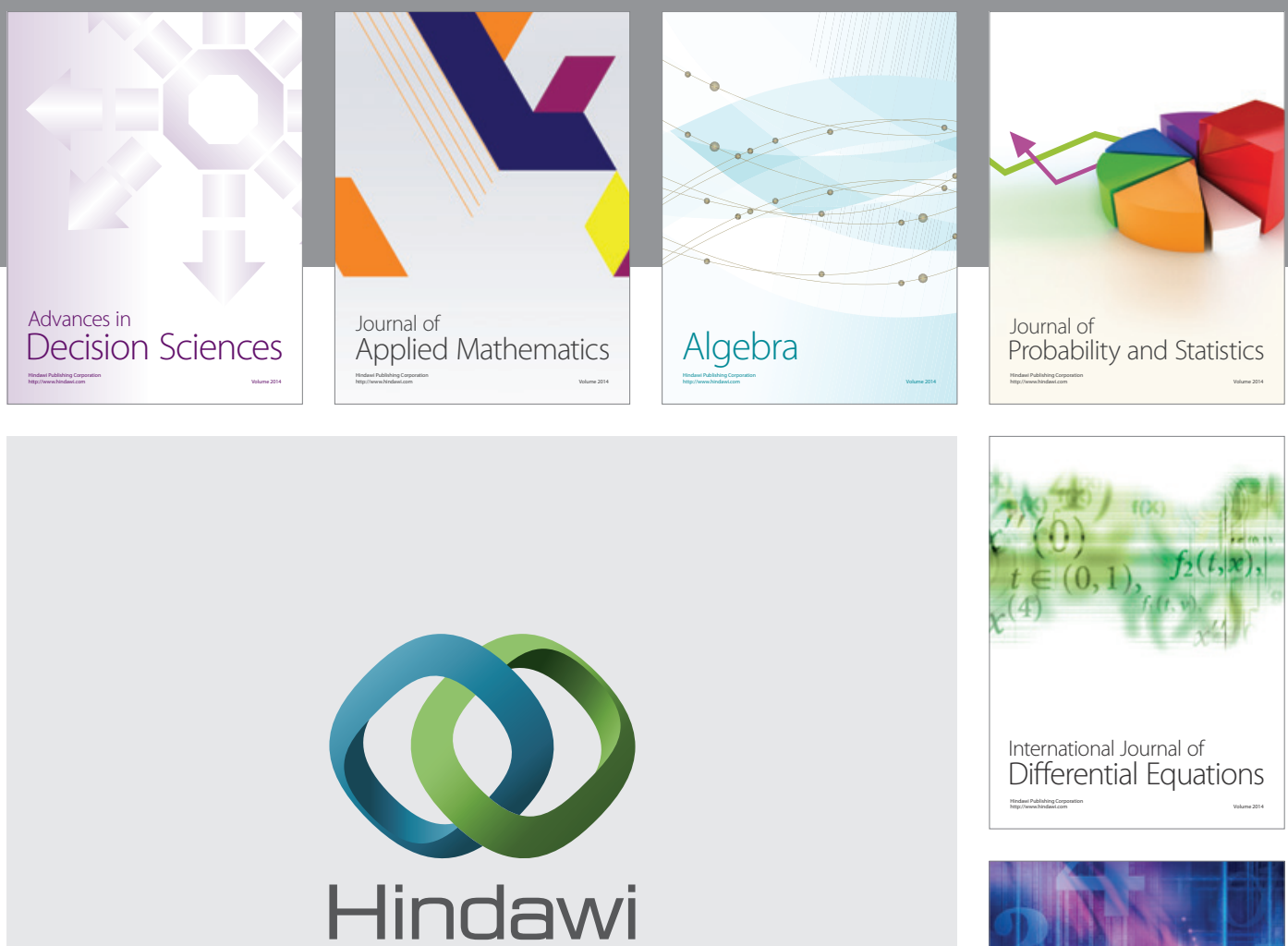

Submit your manuscripts at http://www.hindawi.com
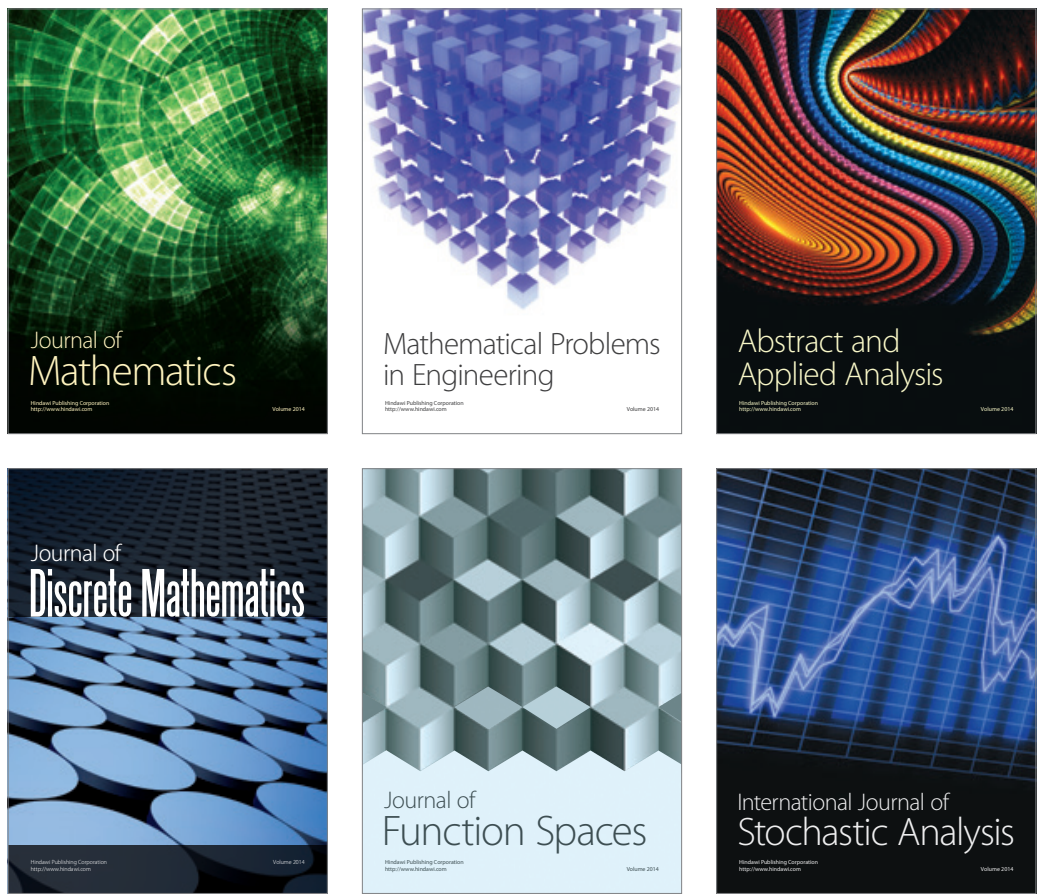

Journal of

Function Spaces

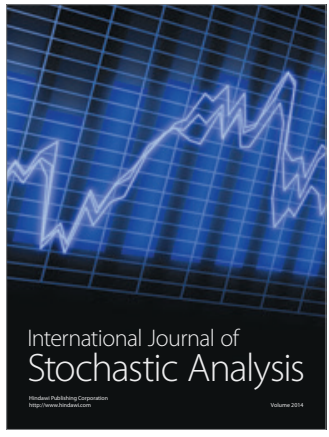

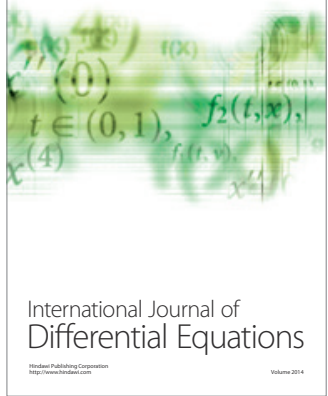
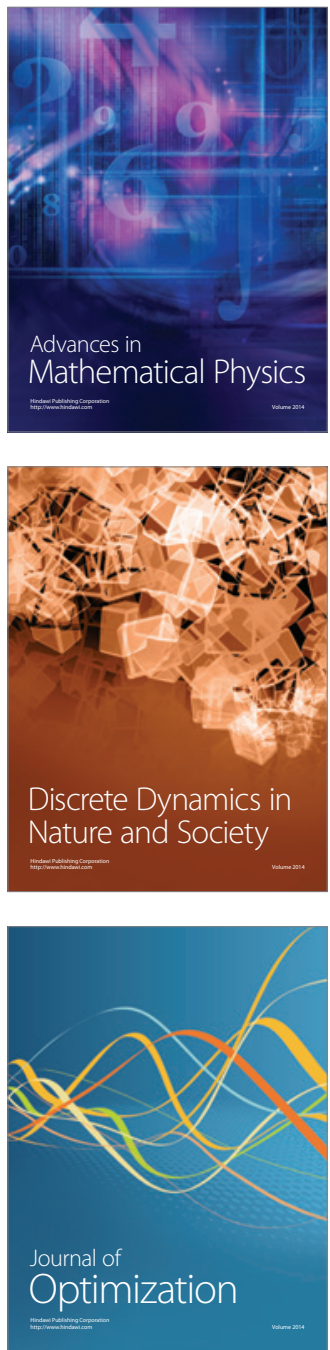